\title{
Drell-Yan measurements at the COMPASS experiment
}

\author{
Marco Meyer-Conde ${ }^{* \dagger}$ \\ University of Illinois at Urbana-Champaign, USA \\ E-mail: marco.meyer@cern.ch
}

In 2015 and 2018 the COMPASS experiment at CERN measured muon-pair production in the Drell-Yan (DY) and J/Psi channels using a $190 \mathrm{GeV} \pi^{-}$beam impinging on a transversely polarized $\mathrm{NH}_{3}$ and unpolarized nuclear targets $\left({ }^{27} \mathrm{Al},{ }^{184} \mathrm{~W}\right)$. Apart from the absolute cross-section measurements for both processes, COMPASS studies also the target spin (in)dependent azimuthal asymmetries in both channels. Assuming the valence quark-antiquark annihilation to be the main production mechanism, one can interpret the azimuthal asymmetries in terms of convolutions of pion and proton Transverse Momentum Dependent (TMD) Parton Distribution Functions (PDFs). The TMD PDFs are universal QCD objects providing a mapping of parton densities in 3D momentum space. Recent results obtained by COMPASS for the DY and $J / \psi$ channels will be discussed along with complementary semi-inclusive DIS (SIDIS) measurements and theoretical predictions.

40th International Conference on High Energy physics - ICHEP2020

July 28 - August 6, 2020

Prague, Czech Republic (virtual meeting)

\footnotetext{
*Speaker

${ }^{\dagger}$ On Behalf of the COMPASS collaboration
} 


\section{Introduction}

The DY process is theoretically well known, which makes it a good probe at COMPASS with its fixed nuclear targets and $\pi^{-}$beam to further our understanding of both (un)polarized nucleon and meson structure. A better constraint on the $\pi$ - quark flavor distributions will significantly contribute to the understanding of the pion structure which currently suffers from a lack of experimental data at fixed target energies.

In the limit of the leading order QCD parton model, the polarised nucleon structure is parametrized by eight TMD PDFs. These functions are universal [1] except for a sign switch of the naive time-reversal odd Sivers and Boer-Mulders TMD PDFs when measured in DY and SIDIS. These functions aim to describe the correlations between the nucleon spin, the parton spin, and the transverse component of the intrinsic parton momentum, $k_{T}$. The study of Transverse Spin-dependent Azimuthal asymmetries (TSAs) is a powerful tool, both in SIDIS and DY, to experimentally access such functions. The TMD factorization theorem [1] allows to decompose these asymmetries as convolutions of TMD PDFs in DY. A similar decomposition applies in SIDIS between TMD PDFs and TMD fragmentation functions. The relation between DY and SIDIS is further discussed in Ref. [2].

A general expression of the $\pi^{-}$induced polarized DY cross section is given in Eq. 1 [3]. At COMPASS, the DY muon pairs are produced by a $190 \mathrm{GeV} / \mathrm{c} \pi^{-}$beam scattering off a transversely polarized proton $\left(\mathrm{NH}_{3}\right)$ targets.

$$
\begin{aligned}
& \frac{d \sigma}{d^{4} q d \Omega} \propto\left\{1+D_{\left[\sin 2 \theta_{\mathrm{CS}]}\right.} A_{U}^{\cos \varphi_{\mathrm{CS}}} \cos \varphi_{\mathrm{CS}}+D_{\left[\sin ^{2} \theta_{\mathrm{CS}}\right]} A_{U}^{\cos 2 \varphi_{\mathrm{CS}}} \cos 2 \varphi_{\mathrm{CS}}\right. \\
& +\left|\vec{S}_{T}\right|\left[D_{\left[1+\cos ^{2} \theta_{\mathrm{CS}]}\right.} A_{T}^{\sin \varphi_{S}} \sin \varphi_{S}\right. \\
& +D_{\left[\sin ^{2} \theta_{\mathrm{CS}}\right]}\left(A_{T}^{\sin \left(2 \varphi_{\mathrm{CS}}-\varphi_{S}\right)} \sin \left(2 \varphi_{\mathrm{CS}}-\varphi_{S}\right)+A_{T}^{\sin \left(2 \varphi_{\mathrm{CS}}+\varphi_{S}\right)} \sin \left(2 \varphi_{\mathrm{CS}}+\varphi_{S}\right)\right) \\
& \left.\left.+D_{\left[\sin 2 \theta_{\mathrm{CS}}\right]}\left(A_{T}^{\sin \left(\varphi_{\mathrm{CS}}-\varphi_{S}\right)} \sin \left(\varphi_{\mathrm{CS}}-\varphi_{S}\right)+A_{T}^{\sin \left(\varphi_{\mathrm{CS}}\right)} \sin \left(\varphi_{C S}+\varphi_{S}\right)\right)\right]\right\}
\end{aligned}
$$

The amplitude coefficients A in the formula denote the TSAs. There is a set of one polar asymmetry, two unpolarized, and five target transverse-spin-dependent azimuthal asymmetries. $A_{(U) T}$ refers to the target transverse polarization (in)dependent amplitudes. The angle definitions (Eq. 1) are given in Fig. 1. Eventually, $D_{\left[f\left(\theta_{\mathrm{CS}}\right)\right]}$ denotes the depolarization factors of the virtual photon in analogy to SIDIS.

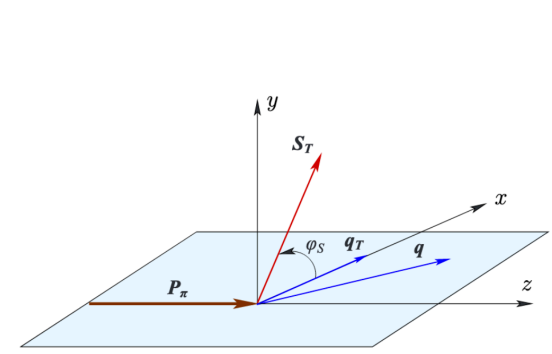

(a)

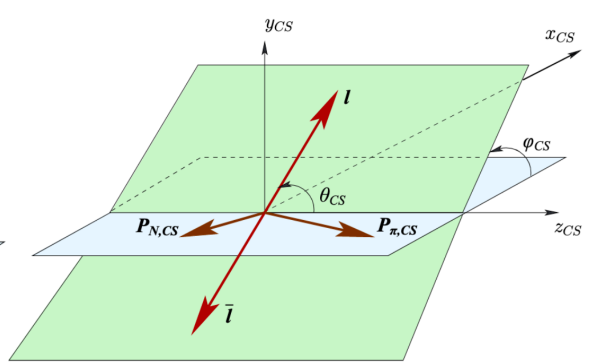

(b)

Figure 1: (a) Definition of the azimuthal angle $\varphi_{S}$ of transverse target spin $S_{T}$ in the target rest frame. (b) Definition of polar and azimuthal angles $\theta_{\mathrm{CS}}$ and $\varphi_{\mathrm{CS}}$ of the lepton momenta in the Collins-Soper frame [3]. 
The DY data offers also valuable information about the nuclear dependence of the DY crosssection by scattering off heavier targets $\left({ }^{27} \mathrm{Al},{ }^{184} \mathrm{~W}\right)$. Therefore, the evaluation of the DY crosssection using COMPASS data might significantly contribute to a better understanding of nuclear effects, such as EMC effects [4] and determine the nuclear corrective factor $R_{i}^{A}$ of the PDF [5]:

$$
\begin{aligned}
f_{i}^{p / A}\left(x, Q^{2}\right)=R_{i}^{A}\left(x, Q^{2}\right) f_{i}^{p}\left(x, Q^{2}\right) & , \text { where } f_{i}^{p} \text { is the free proton PDF } \\
& \text { and } f_{i}^{p / A} \text { the nuclear-corrected PDF }
\end{aligned}
$$

Finally, the $\mathrm{J} / \psi$ channel is also extensively studied as a lot of statistics is available. First constrains on the double $\mathrm{J} / \psi$ production models, namely single/double parton scattering (SPS/DPS) and intrinsic charm (IC) mechanism (Fig. 2) will be further discussed.

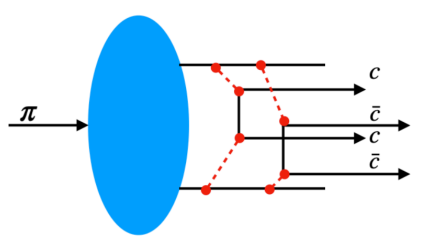

(a)

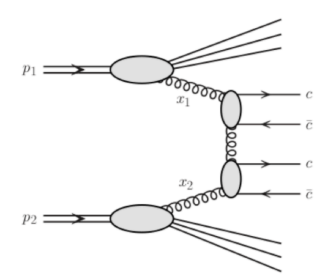

(b)

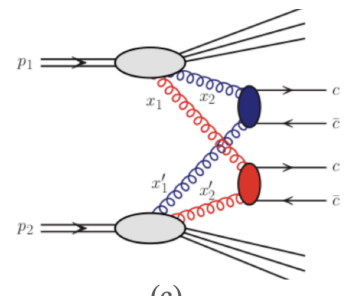

(c)

Figure 2: (a) Intrinsic charm [6]; (b) Single parton scattering model [7]; (c) Double parton scattering [8]

\section{Event analysis}

This Letter is based on the 2015 and 2018 DY data collected at COMPASS, where only 50\% of the 2018 statistics is presented. In 2018, data-taking was divided in 9 periods, each consisting of consecutive weeks with opposite transverse polarizations reversed every two weeks to minimize acceptance effects. The $190 \mathrm{GeV} / \mathrm{c}( \pm 3 \mathrm{GeV} / \mathrm{c})$ hadron beam $\left(96.80 \% \pi^{-}, 2.40 \% \bar{K}, 0.80 \% \bar{p}\right)$, with an average intensity of $7 \times 10^{7} \mathrm{~s}^{-1}$, scattered off two transversely polarized $\mathrm{NH}_{3}$ target cells of opposite polarizations, an aluminum, and a tungsten target (Fig. 3). The selected mass ranges are $4.3 \mathrm{GeV} / \mathrm{c}^{2}<\mathrm{M}_{\mu \mu}<8.5 \mathrm{GeV} / \mathrm{c}^{2}$ for DY and $2.5 \mathrm{GeV} / \mathrm{c}^{2}<\mathrm{M}_{\mu \mu}<4.3 \mathrm{GeV} / \mathrm{c}^{2}$ for $\mathrm{J} / \psi$ (Fig. 4a). Events with $q_{T}<0.5 \mathrm{GeV} / \mathrm{c}$ are removed from the selection to improve the azimuthal resolution. The kinematic phase space probed in $x_{\pi}$ vs $x_{N}$ is shown in Fig. $4 \mathrm{~b}$, where a wide range of the internal structure of the $\pi^{-}$is probed.

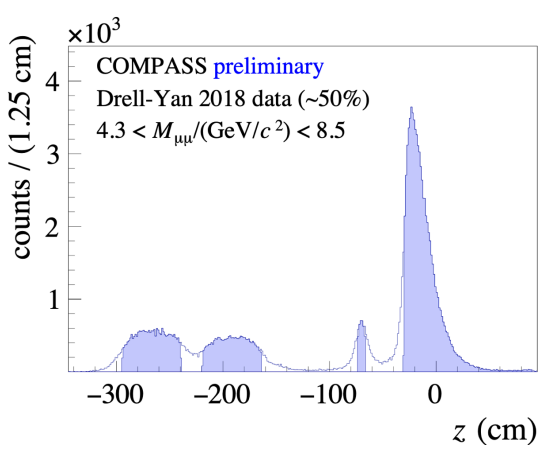

Figure 3: DY vertices in HM range.
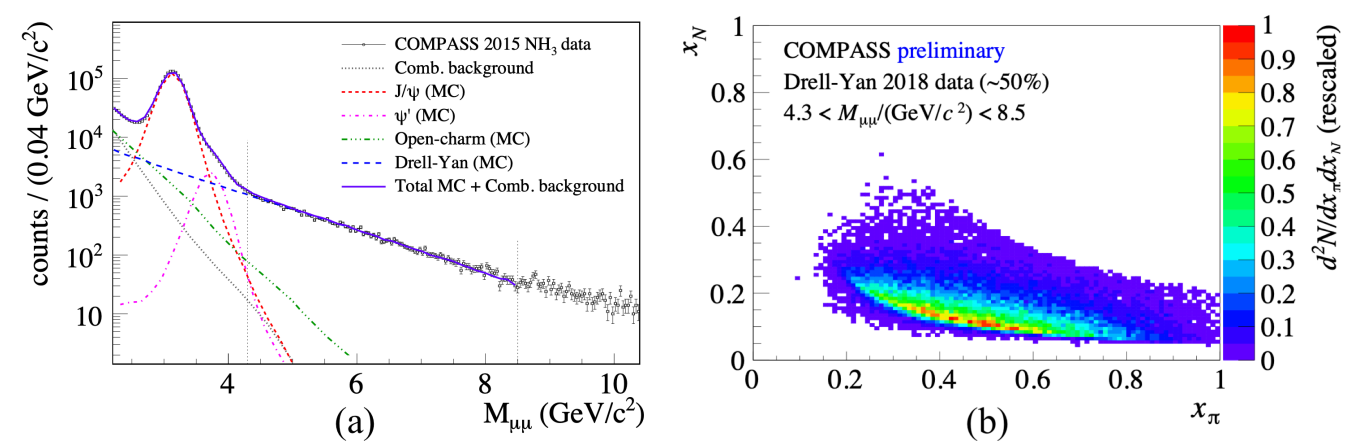

Figure 4: (a) Dimuon mass for $\mathrm{NH}_{3}$ data. (b) $x_{N}$ vs $x_{\pi}$ range probed by COMPASS DY experiment. 


\section{Results and discussions}

\subsection{Polarized Drell-Yan measurements}

The three DY twist-2 TSAs $A_{T}^{\sin \varphi_{S}}, A_{T}^{\sin \left(2 \varphi_{\mathrm{CS}}-\varphi_{S}\right)}, A_{T}^{\sin \left(2 \varphi_{\mathrm{CS}}+\varphi_{S}\right)}$, and the two subleading-twist TSAs $A_{T}^{\sin \left(\varphi_{\mathrm{CS}}-\varphi_{S}\right)}, A_{T}^{\sin \left(\varphi_{\mathrm{CS}}+\varphi_{S}\right)}$ are presented in Fig.5a. In the figure, empty circles are the asymmetries obtained from 2015 data, while filled circles refers to the combined statistical occurrence of 2015 and th preliminary 2018 data. The systematic uncertainties are estimated at about 0.7 times the statistical uncertainties and are indicated in Fig.5a by the filled/empty bands on the left.

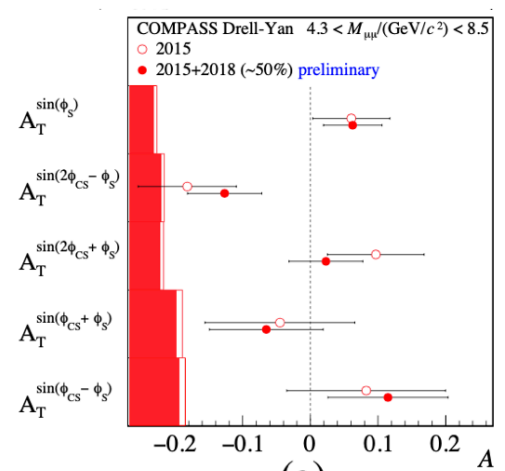

(a)

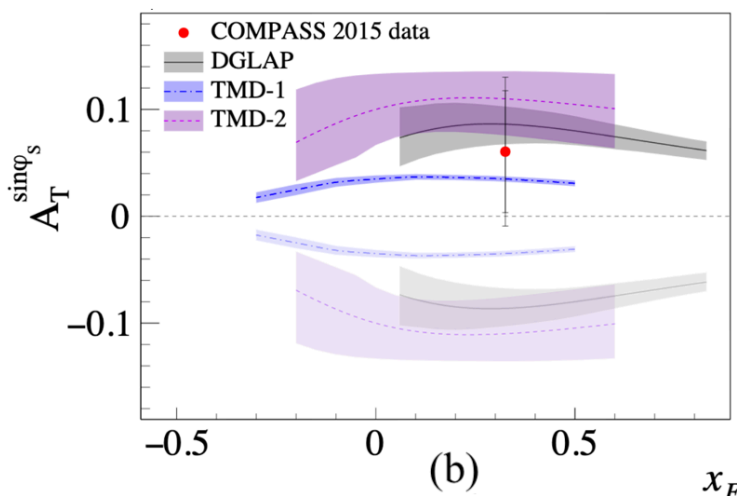

(b)

Figure 5: (a) DY TSAs integrated over the entire kinematic range; (b) The measured mean Sivers asymmetry and the theoretical predictions for different $Q^{2}$ evolution schemes from [9] (DGLAP), [10] (TMD1) and [11] (TMD2). The dark-shaded (light-shaded) predictions are evaluated with (without) the sign-change hypothesis. The error bar represents the total experimental uncertainty and the inner error bar the statistical uncertainty only.

The Sivers asymmetry $A_{T}^{\sin \varphi_{S}}$ in DY is found to be above zero at about one standard deviation and confirms the sign change expected between DY and SIDIS at the 2-sigma level (Fig. 5b, 2015 data only). The TSA $A_{T}^{\sin \left(2 \varphi_{\mathrm{CS}}-\varphi_{S}\right)}$, related to the nucleon transversity TMD PDF, is measured to be below zero about two standard deviations of the total uncertainty and compatible with theoretical models [12]. The pretzelosity TSA $A_{T}^{\sin \left(2 \varphi_{\mathrm{CS}}+\varphi_{S}\right)}$ is found to be compatible with zero when combining the two-year results. Eventually, the remaining subleading-twist TSAs, $A_{T}^{\sin \left(\varphi_{\mathrm{CS}}-\varphi_{S}\right)}$ and $A_{T}^{\sin \left(\varphi_{\mathrm{CS}}-\varphi_{S}\right)}$ are found to be compatible with zero, as expected due to the subleading nature of the effects. The kinematic dependencies for all five DY TSAs are discussed in detail in Ref. [13].

\subsection{Unpolarized Drell-Yan studies}

To quantify the effects of cold nuclear matter (cold QCD) such as parton energy loss and nuclear PDFs, COMPASS studies the nuclear dependence of the DY process by measuring cross-section ratios on nuclear targets. At leading order, the ratio between the DY cross-sections for $\mathrm{NH}_{3}$ (or ${ }^{27} \mathrm{Al}$ ) and ${ }^{184} \mathrm{~W}$ is dominated by the ratio of their valence quarks distributions. In $\pi^{-}$DY collisions, the valence $\bar{u}$-quark in the beam and the valence $u$-quark in the target are

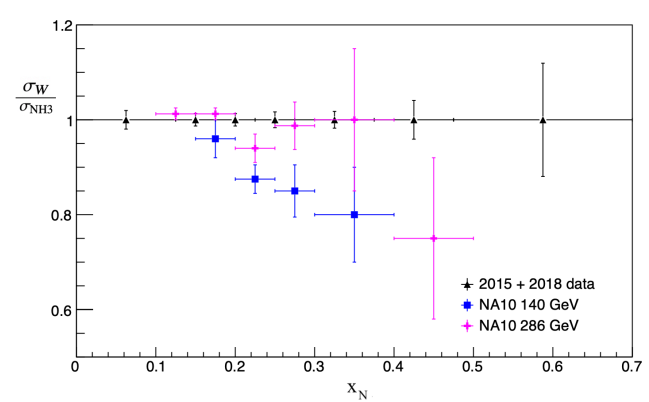

Figure 6: Projected statistical uncertainties of the ratio $\sigma_{W} / \sigma_{\mathrm{NH} 3}$ for $2015+2018 \mathrm{DY}$ data. 
preferentially probed. The projected statistical uncertainty $2015+2018$ for the $\mathrm{W} / \mathrm{NH}_{3}$ ratio is shown Fig. 7 and compared with the existing data from NA10 [14]. The wide range of the COMPASS data is expected to provide enhanced constraints on the effect of the modification of the DY cross-section in nuclear media.

In extenso, the expected statistical uncertainty of the extracted DY cross-section on tungsten is shown Fig. 7. The results are shown in bins of $\sqrt{\tau}(=M / \sqrt{s}): 0.277<\sqrt{\tau}<0.323$ and $0.323<\sqrt{\tau}<0.369$. All the distributions are scaled by the square of center-of-mass energy in order to get energy-independent comparison with the published data from E615 [15] and a MC simulation at NNLO using GRV $\times$ CT14 (green bands).
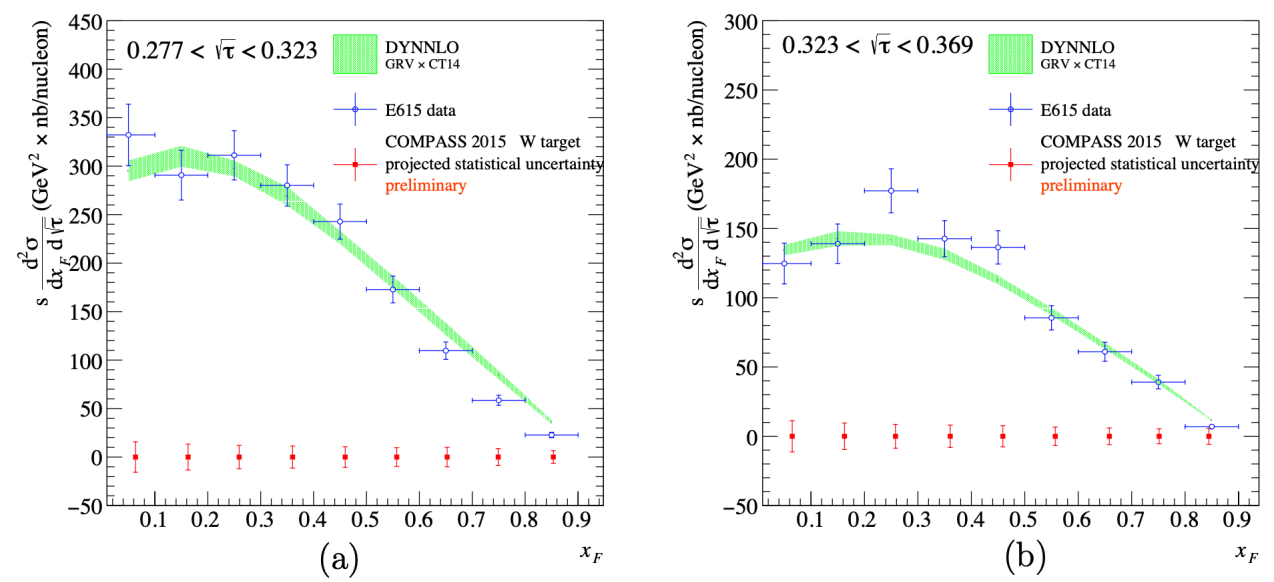

Figure 7: The expected statistical uncertainties of DY differential cross sections on a W target vs. $x_{F}$. (a) $0.277<\tau<0.323$; (b) $0.323<\tau<0.369$

\subsection{Double $\mathbf{J} / \psi$ production study}

The current theoretical developments assume that the only leading production mechanism for double $\mathrm{J} / \psi$ is due to SPS or IC mechanism (Fig. 2). However, some contributions from the DPS and interference between SPS and IC are expected. DPS is expected to be strongly suppressed at fixed target energies. A measurement of double $\mathrm{J} / \psi$ cross-section has been performed in a range around the $\mathrm{J} / \psi$ mass (Fig.8a) on various targets (Eq.3) for $x_{F}>0$. The systematic uncertainties are estimated at about 0.6 times the statistical uncertainties.

$$
\begin{aligned}
\left.\left(\frac{\sigma_{2 J / \psi}}{\sigma_{J / \psi}}\right)\right|_{x_{F}>0} & =\left(1.1 \pm 0.3_{\text {stat }} \pm 0.2_{\text {sys }}\right) .10^{-4} \\
\left.\sigma_{J / \psi J / \psi}\left(\mathrm{NH}_{3}\right)\right|_{x_{F}>0} & =\left(8.8 \pm 2.2_{\text {stat }} \pm 2.4_{\text {sys }}\right) \mathrm{pb} / \text { nucleon } \\
\left.\sigma_{J / \psi J / \psi}(\mathrm{Al})\right|_{x_{F}>0} & =\left(3.4 \pm 4.3_{\text {stat }} \pm 5.8_{\text {sys }}\right) \mathrm{pb} / \text { nucleon } \\
\left.\sigma_{J / \psi J / \psi}(\mathrm{W})\right|_{x_{F}>0} & =\left(14.3 \pm 7.7_{\text {stat }} \pm 4.5_{\text {sys }}\right) \mathrm{pb} / \text { nucleon }
\end{aligned}
$$

Moreover, the SPS contribution appears to be a dominant mechanism, as the contribution of IC mechanism seems negligible in Fig. 8b. Therefore, the upper limit of the model is better constrained at fixed target energies and over a wide kinematic range in $x_{\|}=P_{z} / P_{\text {beam }}$. 


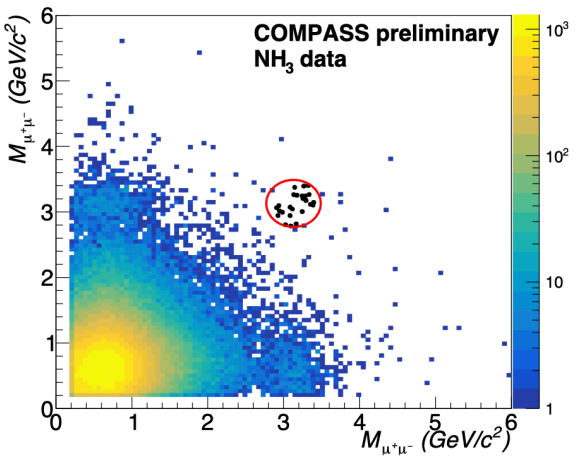

(a)

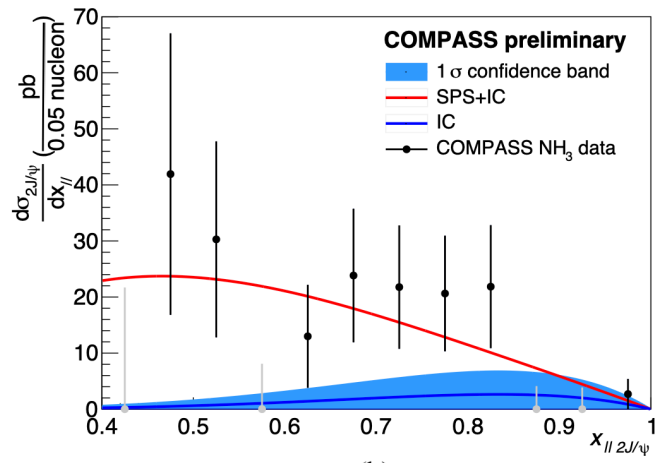

(b)

Figure 8: (a) Two-dimensional mass distribution of events with two muon pairs highlighting the considered double $\mathrm{J} / \psi$ region (red circle: $3.13 \pm 0.18 \mathrm{GeV} / \mathrm{c}^{2}$ ); (b) Double $\mathrm{J} / \psi$ cross-section as a function of $x$ on $\mathrm{NH}_{3}$ target compared with theoretical models including IC and IC+SPS.

\section{References}

[1] J. Collins, Foundations of perturbative QCD. Cambridge University Press, 2013.

[2] B. Parsamyan [COMPASS Collaboration], PoS DIS 2019 (2019) 195.

[3] COMPASS Collaboration, SPSC-P-340, CERN-SPSC-2010-014 (2010).

[4] J.J. Aubert et al., Phys. Lett. B123.3 (1983), pp. 275-278.

[5] Eskola et al., Eur. Phys. J. C1612.05741 (2017)

[6] R. Vogt, S.J. Brodsky, Phys. Lett. B349:569-575 (1995)

[7] R.E. Ecclestone, D.M. Scott Phys. Lett. 120B (1983) 237

[8] V.G.Kartvelishvili, S.M.Esakiya Yad. Fiz. 38 (1983) 722-726

[9] M. Anselmino, M. Boglione, U. D’Alesio, F. Murgia and A. Prokudin, JHEP 1704 (2017) 046.

[10] M. G. Echevarria, A. Idilbi, Z. B. Kang and I. Vitev, Phys. Rev. D89 (2014) 074013.

[11] P. Sun and F. Yuan, Phys. Rev. D88 (2013) 034016.

[12] A. N. Sissakian, O. Yu. Shevchenko, et al., Phys. Part. Nucl. 41 (2010) 64-100.

[13] COMPASS Collaboration, M.Aghasyan et al., Phys. Rev. Lett. 119 (2017) 112002.

[14] NA10 Collaboration, Bordalo P. et al., Phys.Lett.B193 (1987) 368.

[15] E615 Collaboration, J.S.Conway et al. Phys.Rev.D39 (1989), pp. 92-122. 